\title{
A SECOND EIGENVALUE BOUND FOR THE DIRICHLET LAPLACIAN IN HYPERBOLIC SPACE
}

\author{
RAFAEL D. BENGURIA AND HELMUT LINDE
}

\begin{abstract}
Let $\Omega$ be some domain in the hyperbolic space $\mathbb{H}^{n}$ (with $n \geq 2$ ) and $S_{1}$ the geodesic ball that has the same first Dirichlet eigenvalue as $\Omega$. We prove the Payne-Pólya-Weinberger conjecture for $\mathbb{H}^{n}$, i.e., that the second Dirichlet eigenvalue on $\Omega$ is smaller or equal than the second Dirichlet eigenvalue on $S_{1}$.

We also prove that the ratio of the first two eigenvalues on geodesic balls is a decreasing function of the radius.
\end{abstract}

\section{INTRODUCTION}

In the proof of the Payne-Pólya-Weinberger (PPW) conjecture [4, Ashbaugh and one of us showed that the ratio of the first two Dirichlet eigenvalues of the Laplacian operator on a bounded domain in Euclidean space is maximized when the domain is a circle. This result was later generalized to domains that are contained in a hemisphere of $\mathbb{S}^{n}[8]$ and, more recently, to Schrödinger operators in the Euclidean space and to the Dirichlet Laplacian in Gaussian space [18, 10. In the work at hand we prove an analogous result for the hyperbolic space that has already been conjectured in [8]:

Theorem 1.1 (The PPW inequality for $\mathbb{H}^{n}$ ). Let $\Omega \subset \mathbb{H}^{n}$ be an open bounded domain in the hyperbolic space of constant negative curvature $\kappa \equiv-\rho^{-2}$ and call $\lambda_{i}(\Omega, \rho)$ the $i$-th Dirichlet eigenvalue on $\Omega$. If $S_{1} \subset \mathbb{H}^{n}$ is a geodesic ball such that $\lambda_{1}(\Omega, \rho)=\lambda_{1}\left(S_{1}, \rho\right)$ then

$$
\lambda_{2}(\Omega, \rho) \leq \lambda_{2}\left(S_{1}, \rho\right)
$$

with equality if and only if $\Omega$ is a geodesic ball.

For the precise definitions of $\mathbb{H}^{n}$ and the Laplacian operator in it, see the following section.

We remark that the inequality (11) has not the form of the original PPW estimate

$$
\frac{\lambda_{2}(\Omega)}{\lambda_{1}(\Omega)} \leq \frac{\lambda_{2}\left(\Omega^{\star}\right)}{\lambda_{1}\left(\Omega^{\star}\right)}, \quad \Omega \subset \mathbb{R}^{n},\left|\Omega^{\star}\right|=|\Omega|,
$$

where the ratio of the first two eigenvalues on $\Omega$ is compared to that on the spherical rearrangement $\Omega^{\star}$. It has been pointed out in [8] already,

R.B. was supported by FONDECYT project \# 102-0844. H.L. gratefully acknowledges financial support from CONICYT. 
that an estimate in the form (10) is the more natural result for settings where $\lambda_{2} / \lambda_{1}$ on a ball is a non-constant function of the ball's radius. In the case of a domain on a hemisphere, for example, $\lambda_{2} / \lambda_{1}$ on a ball is an increasing function of the radius [8]. But by the Rayleigh-FaberKrahn inequality for spheres [28] the radius of $S_{1}$ is smaller than the one of the spherical rearrangement $\Omega^{\star}$. This means that an estimate in the form (11), interpreted as

$$
\frac{\lambda_{2}(\Omega)}{\lambda_{1}(\Omega)} \leq \frac{\lambda_{2}\left(S_{1}\right)}{\lambda_{1}\left(S_{1}\right)}, \quad \Omega \subset \mathbb{S}^{n},
$$

is stronger than an inequality of the type (2).

On the other hand, we have shown in [10] that $\lambda_{2} / \lambda_{1}$ on balls is a strictly decreasing function of the radius for the Dirichlet Laplacian in the space with a measure of inverted Gaussian density. In this case we can apply an argument from [8] to see that an estimate of the type (2) cannot possibly hold true: Consider a domain $\Omega$ that is constructed by attaching very long and thin tentacles to the ball $B$. Then the first and second eigenvalues of the Laplacian on $\Omega$ are arbitrarily close to the ones on $B$. The spherical rearrangement of $\Omega$ though can be considerably larger than $B$. This means that

$$
\frac{\lambda_{2}(\Omega)}{\lambda_{1}(\Omega)} \approx \frac{\lambda_{2}(B)}{\lambda_{1}(B)}>\frac{\lambda_{2}\left(\Omega^{\star}\right)}{\lambda_{1}\left(\Omega^{\star}\right)}, \quad B, \Omega \subset\left(\mathbb{R}^{n}, e^{r^{2}} \mathrm{~d}^{n} r\right)
$$

clearly ruling out any inequality in the form of (2).

With respect to the behavior of $\lambda_{2} / \lambda_{1}$ on balls, our present case of the hyperbolic space is similar to the one of the inverted-Gaussian space:

Theorem 1.2 (Monotonicity of $\lambda_{2} / \lambda_{1}$ on balls in $\left.\mathbb{H}^{n}\right)$. Let $\lambda_{i}(\theta, \rho)$ be the $i$-th eigenvalue of the Dirichlet-Laplacian on the ball of geodesic radius $\theta$. Then $\lambda_{2}(\theta, \rho) / \lambda_{1}(\theta, \rho)$ is a strictly decreasing function of $\theta$.

Repeating the argument given above for the inverted-Gaussian space, we see that an inequality of the type (2) can not hold true in $\mathbb{H}^{n}$.

Our proof of Theorem 1.1 follows the general lines of [4, though each step has to be modified to fit our special setting. This is how the rest of the present article is organized:

In the following section we summarize briefly how $\mathbb{H}^{n}$ and the differential operators in it are defined in terms of Riemannian geometry. For a comprehensive introduction to the spectral theory of differential operators on Riemannian manifolds we refer the reader to [13].

In Section 3 and Section 4 we will state several results about the first two Dirichlet eigenvalues on geodesic balls in $\mathbb{H}^{n}$. First, to identify the second eigenvalue, we prove a lemma that is somewhat analogous to the Baumgartner-Grosse-Martin inequality [11, 12, 1] for Schrödinger operators in Euclidean space. Second, we prove Theorem 1.2 and some 
other facts about the first two eigenvalues on balls. In contrast to [8] we do not apply perturbation theory to prove the monotonicity of $\lambda_{2} / \lambda_{1}$ on balls of varying radius. Instead we use scaling properties of the eigenvalues in combination with a lemma that is similar to Theorem 1.1. but that compares the eigenvalues on balls in hyperbolic spaces of different curvature. We believe that this is the more natural way to prove Theorem 1.2 and it has the advantage to deliver some additional lemmata as byproducts.

Section 5 shows the bare-bone structure of the proof of Theorem 1.1 which consists as usual [4, 8, in using the gap inequality for $\lambda_{1}$ and $\lambda_{2}$, the choice of suitable test functions and finally the use of rearrangement techniques and monotonicity arguments.

The final four sections are to fill the gaps that are left open in Section 5. First we prove the center of mass' result for $\mathbb{H}^{n}$. Then, in sections [7] and 8 we prove the monotonicity of certain functions $g$ and $B$. In comparison to the Euclidean or spherical case, this part of our proof causes quite a lot of difficulties as can be seen in the corresponding sections.

Finally, in Section 9 a version of Chiti's comparison result is proven, in a slightly simplified way compared to the proof in [4, 8].

\section{Preliminaries about the Laplacian operator in $\mathbb{H}^{n}$}

In this section we briefly summarize how to define the hyperbolic space and the differential operators in it. We follow the definitions of [13, where a more detailed discussion of the subject can be found. We fix a constant negative curvature $\kappa<0$ and set $\rho=(-\kappa)^{-1 / 2}$. Then we realize $\mathbb{H}^{n}$ as the ball $B^{n}(\rho) \subset \mathbb{R}^{n}$ of radius $\rho$ endowed with the metric

$$
d s^{2}=\frac{4\|d x\|^{2}}{\left(1-\|\vec{x} / \rho\|^{2}\right)^{2}},
$$

where $\|\cdot\|$ denotes the Euclidean norm. We define spherical coordinates by writing $\vec{x} \in B^{n}(\rho)$ as

$$
\vec{x}=\rho \tanh (\theta / 2 \rho) \vec{\chi},
$$

where $\vec{\chi} \in \mathbb{S}^{n-1}$ is a vector of the unit sphere and $\theta \in[0, \infty)$. The hyperbolic metric (3) takes in our new coordinates the form

$$
d s^{2}=d \theta^{2}+\rho^{2} \sinh ^{2}(\theta / \rho)\|d \vec{\chi}\|^{2} .
$$

This metric induces an inner product $\langle.,$.$\rangle and norm |\cdot|$ on the tangent space at any point of $\mathbb{H}^{n}$. Each tangent space is isomorphic to $\mathbb{R}^{n}$ and its elements are associated with directional derivatives of $C^{1}$ functions defined on $\mathbb{H}^{n}$.

The Riemannian measure in $\mathbb{H}^{n}$ is given by

$$
\mathrm{d} V=\rho^{n-1} \sinh ^{n-1}(\theta / \rho) \mathrm{d} \theta \mathrm{d} \sigma,
$$


where $\mathrm{d} \sigma$ is the measure on $\mathbb{S}^{n-1}$. We write $(.,$.$) for the scalar product$ in $L^{2}\left(\mathbb{H}^{n}\right)$.

We define the divergence of a vector field $X$ in a point $p$ by

$$
\left.\operatorname{div} X\right|_{p}=\operatorname{trace}\left(\xi \rightarrow \nabla_{\xi} X\right),
$$

where $\xi$ ranges over the tangent space in $p$ and $\nabla$ is the Levi-Civita connection of $\mathbb{H}^{n}$.

Given a function $f \in L^{2}\left(\mathbb{H}^{n}\right)$, we write $Y=\operatorname{Grad} f$ for a vector field $Y$ if

$$
(Y, X)=-(f, \operatorname{div} X)
$$

for all $C^{1}$ vector fields $X$ with compact support in $\mathbb{H}^{n}$. If $f$ is a $C^{1}$ function then

$$
\langle\operatorname{Grad} f, \xi\rangle=\xi f
$$

holds in each point $p$ of $\mathbb{H}^{n}$ and for each tangent vector $\xi$.

As usual, we define the Dirichlet Laplacian $-\Delta_{\rho}$ on some bounded domain $\Omega \subset \mathbb{H}^{n}$ by its quadratic form

$$
D[u]=(\operatorname{Grad} u, \operatorname{Grad} u),
$$

defined on the completion of $C_{0}^{\infty}(\Omega)$ with respect to the norm induced by $D[u]$. It is known that $-\Delta_{\rho}$ is then a positive self-adjoint operator with a pure point spectrum, and as mentioned before we call $\lambda_{i}(\Omega, \rho)$ its $i$-th eigenvalue. In the case $\rho=1$ we will usually leave the second argument away. Recall that in Theorem 1.1 we defined $S_{1}$ to be a geodesic ball such that $\lambda_{1}(\Omega, \rho)=\lambda_{1}\left(S_{1}, \rho\right)$. We chose $S_{1}$ to be centered at the origin of our spherical coordinate system and we will call $\tilde{\theta}$ the geodesic radius of $S_{1}$.

The differential expression of $-\Delta_{\rho}$ is

$$
\begin{aligned}
-\Delta_{\rho} u= & -\sinh ^{1-n}(\theta / \rho) \frac{\partial}{\partial \theta}\left(\sinh ^{n-1}(\theta / \rho) \frac{\partial u}{\partial \theta}\right) \\
& -\rho^{-2} \sinh ^{-2}(\theta / \rho) \Delta_{\mathbb{S}^{n-1}} u,
\end{aligned}
$$

where $\Delta_{\mathbb{S}^{n-1}}$ is the Laplacian on the unit sphere.

The Rayleigh-Ritz characterization of the eigenvalues $\lambda_{i}$ of $-\Delta$ is completely analogous to the Euclidean case: If $u \in L^{2}(\Omega)$ is some function in the domain of $D$ and if $u$ is orthogonal to the first $k-1$ eigenfunctions of $-\Delta$, then we have

$$
\lambda_{k}(\Omega) \leq \frac{D[u]}{(u, u)} .
$$

This Rayleigh-Ritz formula is an important ingredient of our proof. Further, we need some more specific information on the first two eigenvalues of $-\Delta$, which will be provided in the following section. 


\section{IDENTIFying The FiRst TWO DiRichlet EIGENVAlues ON GEODESIC BALLS IN $\mathbb{H}^{n}$}

A standard separation of variables (see, e.g., [13], p.40) shows that the eigenvalues and eigenfunctions of $-\Delta_{\rho}$ on a geodesic ball in $\mathbb{H}^{n}$ of radius $\tilde{\theta}$ are determined by the differential equation

$$
-z^{\prime \prime}(\theta)-\frac{(n-1)}{\rho \tanh (\theta / \rho)} z^{\prime}(\theta)+\frac{l(l+n-2)}{\rho^{2} \sinh ^{2}(\theta / \rho)} z(\theta)=\lambda z(\theta),
$$

where $l=0,1,2, \ldots$, with the boundary conditions $z^{\prime}(0)=0$ (for $\left.l=0\right)$ or $z(\theta) \sim \theta^{l}$ as $\theta \downarrow 0$ (for $l>0$ ) and $z(\tilde{\theta})=0$. Throughout the paper we will use the convention that $z_{l}(\theta)>0$ for $\theta \downarrow 0$. If one defines the left hand side of (7) as the operator $h_{l}$ applied to $z$ (with the boundary conditions incorporated in the definition of the operator), then it is easy to see that $h_{l^{\prime}}>h_{l}$ in the sense of quadratic forms if $l^{\prime}>l$. Consequently, the lowest eigenvalue $\lambda_{1}$ of $-\Delta_{\rho}$ on the geodesic ball is $\lambda_{1}\left(h_{0}\right)$, while the second eigenvalue $\lambda_{2}$ on the geodesic ball must be either $\lambda_{2}\left(h_{0}\right)$ or $\lambda_{1}\left(h_{1}\right)$. We show that the later is the case:

Lemma 3.1. The first eigenvalue of the Dirichlet Laplacian on a geodesic ball in $\mathbb{H}^{n}$ is the first eigenvalue of (7) with $l=0$, while the second eigenvalue on the geodesic ball is the first eigenvalue of (7) with $l=1$. The second eigenvalue is $n$-fold degenerate.

Proof. We will prove the lemma for $\rho=1$. The general case follows directly from the behavior of the eigenvalues under rescaling of $\rho$ (see Lemma 4.1 below).

Assume that $z_{l}$ solves (17) for some fixed $\lambda$. Then one can verify that

$$
z_{l}^{\prime}-l \operatorname{coth} \theta z_{l}
$$

satisfies (17) for $l$ replaced by $l+1$ and

$$
z_{l}^{\prime}+(l+n-2) \operatorname{coth} \theta z_{l}
$$

satisfies (71) for $l$ replaced by $l-1$. From these facts and Frobenius theory it follows that

$$
\begin{aligned}
& z_{l+1}=-z_{l}^{\prime}+l \operatorname{coth} \theta z_{l}, \\
& z_{l-1}=z_{l}^{\prime}+(l+n-2) \operatorname{coth} \theta z_{l} .
\end{aligned}
$$

Putting $l=0$ in (8) we get

$$
z_{1}=-z_{0}^{\prime}
$$

Setting $l=1$ in (9) and multiplying with $\sinh ^{n-1} \theta$ yields

$$
\sinh ^{n-1} \theta z_{0}=\left(\sinh ^{n-1} \theta z_{1}\right)^{\prime} .
$$

By Rolle's theorem, between any two zeros of $z_{0}$ there is a zero of $z_{0}^{\prime}$, and hence of $z_{1}$ since (10) holds. Similarly, between two zeros of $\sinh ^{n-1} \theta z_{1}$ there is a zero of its derivative and hence of $z_{0}$ by (11). Thus for fixed $\lambda>0$ the zeros of $z_{0}$ and $z_{1}$ on $[0, \infty)$ interlace. 
Now consider $z_{0}$ and $z_{1}$ for $\lambda=\lambda_{1}\left(h_{1}\right)$. Then the first positive zero of $z_{1}$ is equal to the radius $\tilde{\theta}$ of our geodesic ball. It is clear by what we have just shown that $z_{0}$ has then exactly one zero in $[0, \tilde{\theta}]$ and the second zero of $z_{0}$ is greater than $\tilde{\theta}$. This and the fact that the positive zeros of any $z_{l}$ are decreasing functions of $\lambda$ show that $\lambda_{2}\left(h_{0}\right)>\lambda_{1}\left(h_{1}\right)$.

The degeneracy of the second eigenvalue on the geodesic ball follows from the details of the separation of variables.

For future reference we state the following lemma.

Lemma 3.2. The solution $z_{0}$ of (7) with $l=0$ and some $\lambda>0$ is strictly decreasing on $\left[0, \theta_{0}\right]$, where $\theta_{0}$ is the first positive zero of $z_{0}$. (Note that by our convention $z_{0}>0$.)

Proof. The function $z_{0}$ satisfies

$$
-\left(\sinh ^{n-1} \theta z_{0}^{\prime}\right)^{\prime}=\lambda \sinh ^{n-1} \theta z_{0}>0 \quad \text { for } \theta \in\left[0, \theta_{0}\right],
$$

which implies that $\sinh ^{n-1} \theta z_{0}^{\prime}$ is decreasing in $\left[0, \theta_{0}\right]$. Hence

$$
\sinh ^{n-1} \theta z_{0}^{\prime}<\left.\left(\sinh ^{n-1} \theta z_{0}^{\prime}\right)\right|_{\theta=0}=0,
$$

which proves the Lemma.

\section{Proof of Theorem 1.2}

To prove Theorem 1.2 we need the following lemmata which contain some basic information on the behavior of the first two eigenvalues of $-\Delta_{\rho}$ on geodesic balls.

Lemma 4.1. Using the notation introduced in Section [2, we have for any $c>0, \theta_{0}>0$ and $\rho>0$

$$
\lambda_{i}\left(c \theta_{0}, c \rho\right)=c^{-2} \lambda_{i}\left(\theta_{0}, \rho\right) .
$$

If $z_{i}(\theta)$ is the radial part of the $i$-th eigenfunction of $-\Delta_{\rho}$ on the ball of geodesic radius $\theta_{0}$, then $z_{i}(\theta / c)$ is the radial part of the $i$-th eigenfunction of $-\Delta_{c \rho}$ on the ball of radius $c \theta_{0}$.

The proof of Lemma 4.1 is straight-forward if one replaces $\theta$ in (7) by $\theta / c$ and multiplies both sides with $c^{2}$ afterwards.

Lemma 4.2. The first eigenvalue $\lambda_{1}\left(\theta_{0}, \rho\right)$ of $-\Delta_{\rho}$ on a geodesic ball of radius $\theta_{0}$ is a strictly decreasing function of $\theta_{0}$ and $\rho$, respectively.

Lemma 4.3. Let $0<\rho_{1}<\rho_{2}$ and $\theta_{1}, \theta_{2}$ be such that

$$
\lambda_{1}\left(\theta_{1}, \rho_{1}\right)=\lambda_{1}\left(\theta_{2}, \rho_{2}\right) .
$$

Then $\theta_{1}>\theta_{2}$ and

$$
\lambda_{2}\left(\theta_{1}, \rho_{1}\right) \leq \lambda_{2}\left(\theta_{2}, \rho_{2}\right)
$$


Proof of Lemma 4.2. By setting $z=(\rho \sinh (\theta / \rho))^{(1-n) / 2} v$ one can check that for $l=0$ equation (7) is associated with the one-dimensional Schrödinger operator

$$
H_{0}=-\frac{d^{2}}{d \theta^{2}}+\frac{n-1}{4}\left(\frac{n-1}{\rho^{2}}+\frac{n-3}{\rho^{2} \sinh ^{2}(\theta / \rho)}\right),
$$

acting on $L^{2}\left(\left(0, \theta_{0}\right), d \theta\right)$ with Dirichlet boundary conditions imposed at 0 and $\theta_{0}$. We rewrite the potential term of $H_{0}$ as

$$
V=\frac{(n-1)^{2}}{4 \rho^{2} \tanh ^{2}(\theta / \rho)}-\frac{2}{\rho^{2} \sinh ^{2}(\theta / \rho)},
$$

where it can be checked easily that each of the two summands is strictly decreasing in $\rho$. Thus $\lambda_{1}\left(\theta_{0}, \rho\right)$ is a strictly decreasing function of $\rho$ and, by domain monotonicity, in $\theta_{0}$.

Proof of Lemma 4.3. Let $B_{1}$ and $B_{2}$ be balls of geodesic radii $\theta_{1}, \theta_{2}$ in the hyperbolic spaces of curvature $\kappa_{1}=-\rho_{1}^{-2}$ and $\kappa_{2}=-\rho_{2}^{-2}$, respectively. We call $y_{0}(\theta)$ the first eigenfunction of $-\Delta_{\rho_{1}}$ on $B_{1}, z_{0}(\theta)$ the first eigenfunction of $-\Delta_{\rho_{2}}$ on $B_{2}$ and $z_{1}(\theta)$ the radial part of the second eigenfunction of $-\Delta_{\rho_{2}}$ on $B_{2}$. Keep in mind Lemma 3.1 and our convention to choose eigenfunctions like $y_{0}, z_{0}$ and $z_{1}$ positive. We will assume $y_{0}$ and $z_{0}$ to be normalized to one in $L^{2}\left(B_{1}, \mathrm{~d} V_{1}\right)$ and $L_{2}\left(B_{2}, \mathrm{~d} V_{2}\right)$, respectively. By $\mathrm{d} V_{1}$ and $\mathrm{d} V_{2}$ we denote the hyperbolic measures corresponding to the curvatures $\kappa_{1}$ and $\kappa_{2}$.

Because $\rho_{1}<\rho_{2}$ and because the first eigenvalues on $B_{1}$ and $B_{2}$ are equal, we can conclude from Lemma 4.2 that $\theta_{2}<\theta_{1}$.

In the same way as below in Section [5, with the only difference that $\rho$ is arbitrary and that the 'center of mass' theorem is trivial by symmetry, we derive the gap formula

$$
\lambda_{2}\left(\theta_{1}, \rho_{1}\right)-\lambda_{1}\left(\theta_{1}, \rho_{1}\right) \leq \frac{\int_{B_{1}} y_{0}^{2}(\theta) B_{1}(\theta) \mathrm{d} V_{1}}{\int_{B_{1}} y_{0}^{2}(\theta) g(\theta)^{2} \mathrm{~d} V_{1}}
$$

with

$$
B_{1}(\theta)=\left(\frac{\partial g}{\partial \theta}\right)^{2}+\frac{n-1}{\rho_{1}^{2} \sinh ^{2}\left(\theta / \rho_{1}\right)} g^{2}(\theta) .
$$

We chose $g(\theta)$ to be

$$
g(\theta)= \begin{cases}\frac{z_{1}(\theta)}{z_{0}(\theta)} & \text { for } \theta \in\left[0, \theta_{2}\right) \\ \lim _{\theta \uparrow \theta_{2}} g(\theta) & \text { for } \theta \in\left[\theta_{2}, \theta_{1}\right)\end{cases}
$$

It will be proven in Section 7 that $g$ is an increasing function if $\rho_{1}=1$. Using Lemma 4.1 one can see easily that $g$ is then increasing for any $\rho_{1}>0$. In almost the same way as in Section [7 one can then also show that $B_{1}$ is decreasing for any $\rho_{1}>0$. 
In what follows it turns out to be more convenient to write (14) as

$$
\lambda_{2}\left(\theta_{1}, \rho_{1}\right)-\lambda_{1}\left(\theta_{1}, \rho_{1}\right) \leq \frac{\int_{0}^{\theta_{1}} y_{0}^{2}(\theta) B_{1}(\theta) A_{\rho_{1}}^{\prime}(\theta) \mathrm{d} \theta}{\int_{0}^{\theta_{1}} y_{0}^{2}(\theta) g(\theta)^{2} A_{\rho_{1}}^{\prime}(\theta) \mathrm{d} \theta}
$$

where

$$
A_{\rho}(\theta)=n C_{n} \int_{0}^{\theta} \rho^{n-1} \sinh ^{n-1}\left(\theta^{\prime} / \rho\right) \mathrm{d} \theta^{\prime}
$$

is the measure of a ball of geodesic radius $\theta$ and curvature $\kappa=-\rho^{2}$.

Fact 4.1. For any $\gamma>0$ the functions $y_{0}(\theta)$ and $\gamma z_{0}(\theta)$ do not intersect more than once on $\left[0, \theta_{1}\right]$ ( setting $z_{0}(\theta)=0$ for $\theta>\theta_{2}$ ).

Proof. We consider the functions

$$
p_{1}(\theta)=\frac{y_{0}^{\prime}(\theta)}{y(\theta)} \quad \text { and } \quad p_{2}(\theta)=\frac{z_{0}^{\prime}(\theta)}{z_{0}(\theta)} .
$$

Let us assume that $y_{0}$ and $\gamma z_{0}$ intersect more than once. Then, because both functions are positive and continuous, at one of these intersections (let's say at $\left.\theta=\theta_{3}\right)$ we have $p_{2}\left(\theta_{3}\right) \geq p_{1}\left(\theta_{3}\right)$. At $\theta_{2}$ the function $p_{2}$ goes to minus infinity, while $p_{1}$ remains finite. This means that there must be some $\theta_{4} \in\left[\theta_{3}, \theta_{2}\right)$ such that $p_{2}\left(\theta_{4}\right)=p_{1}\left(\theta_{4}\right)$ and $p_{2}^{\prime}\left(\theta_{4}\right) \leq p_{1}^{\prime}\left(\theta_{4}\right)$

Using (7) we can derive the Riccati equation

$$
p_{i}^{\prime}(\theta)=-p_{i}^{2}(\theta)-\frac{n-1}{\rho_{i} \tanh \left(\theta / \rho_{i}\right)} p_{i}(\theta)-\lambda_{1} \quad(i=1,2) .
$$

Evaluated at $\theta=\theta_{4}$, this equation tells us that

$$
\begin{aligned}
p_{2}^{\prime}\left(\theta_{4}\right)-p_{1}^{\prime}\left(\theta_{4}\right) & =p_{1}\left(\theta_{4}\right)(n-1)\left(\frac{1}{\rho_{1} \tanh \left(\theta / \rho_{1}\right)}-\frac{1}{\rho_{2} \operatorname{coth}\left(\theta / \rho_{2}\right)}\right) \\
& >0
\end{aligned}
$$

which is a contradiction to what has been said before and thus proves the lemma.

Fact 4.2. There is a $\theta_{5} \in\left(0, \theta_{2}\right)$ such that

$$
\begin{array}{ll}
z_{0}(\theta) A_{\rho_{2}}^{\prime}(\theta) \geq y_{0}(\theta) A_{\rho_{1}}^{\prime}(\theta) & \text { for } 0<\theta<\theta_{5}, \\
z_{0}(\theta) A_{\rho_{2}}^{\prime}(\theta) \leq y_{0}(\theta) A_{\rho_{1}}^{\prime}(\theta) & \text { for } \theta_{5}<\theta<\theta_{1} .
\end{array}
$$

Proof. By the normalization of $y_{0}$ and $z_{0}$ it is clear that the two functions $z_{0} A_{\rho_{2}}^{\prime}$ and $y_{0} A_{\rho_{1}}^{\prime}$ intersect at least once on $\left(0, \theta_{2}\right)$. This means that also $z_{0} A_{\rho_{2}}^{\prime} / A_{\rho_{1}}^{\prime}$ and $y_{0}$ intersect at least once, say in $\theta_{5}$. Then by Fact 4.1 the functions

$$
z_{0}(\theta) A_{\rho_{2}}^{\prime}\left(\theta_{5}\right) / A_{\rho_{1}}^{\prime}\left(\theta_{5}\right) \quad \text { and } \quad y_{0}(\theta)
$$

intersect exactly once on $\left(0, \theta_{1}\right)$, and because $y_{0}\left(\theta_{2}\right)>z_{0}\left(\theta_{2}\right)=0$ we know that

$$
\begin{array}{ll}
z_{0}(\theta) A_{\rho_{2}}^{\prime}\left(\theta_{5}\right) / A_{\rho_{1}}^{\prime}\left(\theta_{5}\right) \geq y_{0}(\theta) & \text { for } 0<\theta<\theta_{5}, \\
z_{0}(\theta) A_{\rho_{2}}^{\prime}\left(\theta_{5}\right) / A_{\rho_{1}}^{\prime}\left(\theta_{5}\right) \leq y_{0}(\theta) & \text { for } \theta_{5}<\theta<\theta_{1} .
\end{array}
$$


Finally we note that

$$
A_{\rho_{2}}^{\prime} / A_{\rho_{1}}^{\prime}=\left(\frac{\rho_{2} \sinh \left(\theta / \rho_{2}\right)}{\rho_{1} \sinh \left(\theta / \rho_{1}\right)}\right)^{n-1}
$$

is a decreasing function, such that (18) follows from (19).

Using Fact 4.2 and the monotonicity properties of $g$ and $B$, we can derive from (17) that

$$
\lambda_{2}\left(\theta_{1}, \rho_{1}\right)-\lambda_{1}\left(\theta_{1}, \rho_{1}\right) \leq \frac{\int_{0}^{\theta_{1}} z_{0}^{2}(\theta) B_{1}(\theta) A_{\rho_{2}}^{\prime}(\theta) \mathrm{d} \theta}{\int_{0}^{\theta_{1}} z_{0}^{2}(\theta) g(\theta)^{2} A_{\rho_{2}}^{\prime}(\theta) \mathrm{d} \theta}
$$

If we define $B_{2}$ in the same way as $B_{1}$ in (15), just replacing $\rho_{1}$ by $\rho_{2}$, we have $B_{2}(\theta)>B_{1}(\theta)$ and therefore

$$
\lambda_{2}\left(\theta_{1}, \rho_{1}\right)-\lambda_{1}\left(\theta_{1}, \rho_{1}\right) \leq \frac{\int_{0}^{\theta_{1}} z_{0}^{2}(\theta) B_{2}(\theta) A_{\rho_{2}}^{\prime}(\theta) \mathrm{d} \theta}{\int_{0}^{\theta_{1}} z_{0}^{2}(\theta) g(\theta)^{2} A_{\rho_{2}}^{\prime}(\theta) \mathrm{d} \theta}=\lambda_{2}\left(\theta_{2}, \rho_{2}\right)-\lambda_{1}\left(\theta_{2}, \rho_{2}\right),
$$

proving Lemma 4.3.

Proof of Theorem 1.2. We fix some $0<\theta_{1}<\theta_{2}$ and some $\rho>0$ and define the function $\Theta(c)$ for $0<c \leq 1$ implicitly by

$$
\lambda_{1}(\Theta(c), \rho)=\lambda_{1}\left(c \theta_{2}, c \rho\right) .
$$

Then $\Theta$ is well defined (by Lemma 4.2) and continuous, $\Theta(1)=\theta_{2}$ and $\Theta(c \rightarrow 0)=0$. We can therefore chose $c_{0} \in(0,1)$ such that $\Theta\left(c_{0}\right)=\theta_{1}$. Theorem 1.2 is now proven by the following chain of (in)equalities:

$$
\begin{aligned}
\frac{\lambda_{2}\left(\theta_{1}, \rho\right)}{\lambda_{1}\left(\theta_{1}, \rho\right)} & =\frac{\lambda_{2}\left(\Theta\left(c_{0}\right), \rho\right)}{\lambda_{1}\left(\Theta\left(c_{0}\right), \rho\right)} \\
& \geq \frac{\lambda_{2}\left(c_{0} \theta_{2}, c_{0} \rho\right)}{\lambda_{1}\left(c_{0} \theta_{2}, c_{0} \rho\right)}=\frac{c_{0}^{2} \lambda_{2}\left(\theta_{2}, \rho\right)}{c_{0}^{2} \lambda_{1}\left(\theta_{2}, \rho\right)}=\frac{\lambda_{2}\left(\theta_{2}, \rho\right)}{\lambda_{1}\left(\theta_{2}, \rho\right)}
\end{aligned}
$$

Here the first step is just the definition of $c_{0}$, the second step follows from Lemma 4.3 and the third one from Lemma 4.1.

\section{Proof of TheOrem 1.1}

Proof of Theorem [1.1. First we argue that it is sufficient to prove the theorem for the case $\rho=1$ : Using the differential expression (6) of $-\Delta_{\rho}$ one can check that if $u(\theta, \vec{\chi})$ is some eigenfunction of $-\Delta_{\rho}$ corresponding to the eigenvalue $\lambda$, then $\tilde{u}(\theta, \vec{\chi})=u(\rho \theta, \vec{\chi})$ is an eigenfunction of $-\Delta_{1}$ corresponding to the eigenvalue $\tilde{\lambda}=\lambda \rho^{2}$. One can convince oneself that it is therefore sufficient to prove Theorem 1.1 for $\rho=1$.

We call $u_{1}$ the first eigenfunction of $-\Delta$ on $\Omega$. Further we recall that $S_{1}$ is a geodesic ball in $\mathbb{H}^{n}$ such that $\lambda_{1}(\Omega)=\lambda_{1}\left(S_{1}\right)$ and we write $z_{0}(\theta)$ for the corresponding eigenfunction. By Lemma 3.1 the second eigenvalue $\lambda_{2}\left(S_{1}\right)$ is $n$-fold degenerate and the corresponding eigenspace 
is spanned by the functions $z_{1}(\theta) \chi_{k}$, where $z_{1}$ is the solution of (17) for $l=1$.

Let $P \neq 0$ be a function on $\Omega$ such that that $P u_{1}$ is in the domain of $D$ as defined in (5) and

$$
\int_{\Omega} P u_{1}^{2} \mathrm{~d} V=0
$$

Then by the Rayleigh-Ritz theorem we have the estimate

$$
\begin{aligned}
\lambda_{2}(\Omega)-\lambda_{1}(\Omega) & =\frac{D\left[P u_{1}\right]}{\left(P u_{1}, P u_{1}\right)}-\lambda_{1} \\
& =\left(P u_{1}, P u_{1}\right)^{-1} \int_{\Omega}\left(\left\langle\operatorname{Grad} P u_{1}, \text { Grad } P u_{1}\right\rangle-\lambda_{1} P^{2} u_{1}^{2}\right) \mathrm{d} V
\end{aligned}
$$

Using (5) and the $\mathbb{H}^{n}$ generalizations of well known formulas for differential operators in the Euclidean space [13, we find the gap inequality

$$
\lambda_{2}(\Omega)-\lambda_{1}(\Omega) \leq \frac{\int_{\Omega} u_{1}^{2}\langle\operatorname{Grad} P, \operatorname{Grad} P\rangle \mathrm{d} V}{\int_{\Omega} P^{2} u_{1}^{2} \mathrm{~d} V} .
$$

We do not only chose only one function $P$ but rather a set of $n$ functions, namely

$$
P_{i}(\theta, \vec{\chi}):=g(\theta) \chi_{i}
$$

with

$$
g(\theta)= \begin{cases}\frac{z_{1}(\theta)}{z_{0}(\theta)} & \text { for } \theta \in[0, \tilde{\theta}), \\ \lim _{\theta \uparrow \tilde{\theta}} g(\theta) & \text { for } \theta \geq \tilde{\theta}\end{cases}
$$

Recall that $\tilde{\theta}$ is the geodesic radius of $S_{1}$ and that by convention $z_{0}$ and $z_{1}$ are positive. With this choice of the $P_{i}$ and by our 'center of mass' theorem (see Section 6) it is always possible to shift $\Omega$ in $\mathbb{H}^{n}$ such that the condition (23) is met.

We calculate that

$$
\begin{aligned}
\sum_{i=1}^{n} P_{i}^{2}(\theta, \vec{\chi}) & =g^{2}(\theta) \\
\sum_{i=1}^{n}\left\langle\operatorname{Grad} P_{i}, \operatorname{Grad} P_{i}\right\rangle & =\left(\frac{\partial g}{\partial \theta}\right)^{2}+(n-1) \sinh ^{-2}(\theta) g^{2}(\theta) .
\end{aligned}
$$

We multiply inequality (24) by $\int_{\Omega} P^{2} u_{1}^{2} \mathrm{~d} V$ and then sum up over $i=$ $1, \ldots, n$ to obtain

$$
\lambda_{2}(\Omega)-\lambda_{1}(\Omega) \leq \frac{\int_{\Omega} u_{1}^{2}(\theta, \vec{\chi}) B(\theta) \mathrm{d} V}{\int_{\Omega} u_{1}^{2}(\theta, \vec{\chi}) g^{2}(\theta) \mathrm{d} V}
$$

with

$$
B(\theta)=g^{\prime}(\theta)^{2}+(n-1) \sinh ^{-2}(\theta) g^{2}(\theta) .
$$


To conclude the proof of Theorem 1.1] we need the following two chains of inequalities:

$$
\begin{aligned}
(28) \int_{\Omega} u_{1}^{2}(\theta, \vec{\chi}) B(\theta) \mathrm{d} V & \leq \int_{\Omega^{\star}} u_{1}^{\star}(\theta)^{2} B^{\star}(\theta) \mathrm{d} V \\
& \leq \int_{\Omega^{\star}} u_{1}^{\star}(\theta)^{2} B(\theta) \mathrm{d} V \leq \int_{S_{1}} z_{0}^{2}(\theta) B(\theta) \mathrm{d} V
\end{aligned}
$$

and

$$
\begin{aligned}
(29) \int_{\Omega} u_{1}^{2}(\theta, \vec{\chi}) g(\theta)^{2} \mathrm{~d} V & \geq \int_{\Omega^{\star}} u_{1}^{\star}(\theta)^{2} g_{\star}(\theta)^{2} \mathrm{~d} V \\
& \geq \int_{\Omega^{\star}} u_{1}^{\star}(\theta)^{2} g(\theta)^{2} \mathrm{~d} V \geq \int_{S_{1}} z_{0}^{2}(\theta) g^{2}(\theta) \mathrm{d} V
\end{aligned}
$$

Here we assume that $z_{0}$ is normalized such that $\int_{\Omega} u_{1}^{2} \mathrm{~d} V=\int_{S_{1}} z_{0}^{2} \mathrm{~d} V$. With a star we denote the spherical rearrangements of functions, i.e., $f^{\star}$ is the spherical decreasing rearrangement of some function $f$ and $f_{\star}$ the increasing one (for more details see Section 9). In each of (28) and (29) the first inequality follows directly from the properties of rearrangements 29]. The second inequality is true because of the monotonicity properties of $g$ and $B$, that will be proven in Section 7 below. The third inequality finally follows from our version of Chiti's comparison result that will be presented in Section 9, and from the monotonicity properties of $g$ and $B$ again. Finally, from (26), (28) and (29) we obtain

$$
\lambda_{2}(\Omega)-\lambda_{1}(\Omega) \leq \frac{\int_{S_{1}} z_{0}^{2}(\theta) B(\theta) \mathrm{d} V}{\int_{S_{1}} z_{0}^{2}(\theta) g^{2}(\theta) \mathrm{d} V}=\lambda_{2}(\Omega)-\lambda_{1}\left(S_{1}\right) .
$$

With $\lambda_{1}(\Omega)=\lambda_{1}\left(S_{1}\right)$ this yields

$$
\lambda_{2}(\Omega) \leq \lambda_{2}\left(S_{1}\right),
$$

proving Theorem 1.1. (It is easy to check that equality in (31) holds if and only if $\Omega$ itself is a geodesic ball.)

\section{6. 'Center of MASS' RESUlt FOR DOMAINS IN $\mathbb{H}^{n}$}

In this section we will show that $\Omega$ can always be shifted in $\mathbb{H}^{n}$ such that the condition (23) is fulfilled. Let us first make a remark though on the notion of 'shifting' in $\mathbb{H}^{n}$. It is well known (see, e.g., 13, p. 37) that the metric of $\mathbb{H}^{n}$ has always the form (4), no matter which point of $\mathbb{H}^{n}$ we chose as the origin of our coordinate system. This can most easily be seen in the Minkovski space realization of $\mathbb{H}^{n}$ : We define

$$
\tilde{\mathbb{H}}^{n}:=\left\{\vec{y} \in \mathbb{R}_{1}^{n}: y_{1}^{2}+\cdots+y_{n}^{2}-y_{n+1}^{2}=-1\right\}
$$

and endow it with the restriction of the Minkovski metric in $\mathbb{R}_{1}^{n}$ to $\tilde{\mathbb{H}}^{n}$. This makes $\mathbb{H}^{n}$ and $\tilde{\mathbb{H}}^{n}$ isometric spaces and an example of an isometry 
between them is

$$
I: \mathbb{H}^{n} \rightarrow \tilde{\mathbb{H}}^{n}, \vec{x}=\tanh \frac{\theta}{2} \vec{\chi} \rightarrow \vec{y}=(\sinh \theta \vec{\chi}, \cosh \theta)
$$

It is further known that each Lorentz transformation in $\mathbb{R}_{1}^{n}$ induces an isometry of $\tilde{\mathbb{H}}^{n}$ onto itself, the hyperbolic analog of shifts and rotations in the Euclidean space. The group of these transformations has the transitivity property, i.e., for any two points $p_{1}, p_{2} \in \tilde{\mathbb{H}}^{n}$ there exists a Lorentz transformation that maps $p_{1}$ on $p_{2}$.

Every Lorentz transformation $R$ on $\tilde{\mathbb{H}}^{n}$ induces an isometry $I R I^{-1}$ on $\mathbb{H}^{n}$. Especially, if we subject our domain $\Omega$ to such a transformation (which we call a 'shift' of $\Omega$ ), the eigenvalues of the Laplcian don't change.

Theorem 6.1 ('Center of Mass'). Let $g(\theta)$ be a positive continuous function on $[0, \infty)$ and $P_{i}(\vec{x})=\chi_{i} g(\theta)$. Then one can shift $\Omega$ (and $u_{1}$ with it) such that

$$
\int_{\Omega} P_{i}(\theta, \vec{\chi}) u_{1}^{2}(\theta, \vec{\chi}) \mathrm{d} V=0 \quad \text { for all } i=1, \ldots, n .
$$

Proof. We will prove Theorem 6.1] in the Minkovski space representation of $\mathbb{H}^{n}$. Let $\tilde{\Omega}=I(\Omega), \tilde{u}_{1}(\vec{y})=u_{1}\left(I^{-1}(\vec{y})\right)$ and $\tilde{P}_{i}(\vec{y})=P_{i}\left(I^{-1}(\vec{y})\right)$. Then

$$
\int_{\Omega} P_{i}(\vec{x}) u_{1}^{2}(\vec{x}) \mathrm{d} V=\int_{\tilde{\Omega}} \tilde{P}_{i}(\vec{y}) \tilde{u}_{1}^{2}(\vec{y}) \mathrm{d} \tilde{V} .
$$

Because it doesn't matter whether we shift $\Omega$ and $u_{1}$ or $P_{i}$, we only have to show that there is some Lorentz transformation $R$ such that

$$
\int_{\tilde{\Omega}} \tilde{P}_{i}(R \vec{y}) \tilde{u}_{1}^{2}(\vec{y}) \mathrm{d} \tilde{V}=0 .
$$

Let $\Theta(\vec{z}, \vec{y})$ be the $\theta$ coordinate of $\vec{y}$ after a Lorentz transformation that maps $\vec{z}$ to $(0, \ldots, 0,1)$ and set

$$
\vec{v}(\vec{z})=\int_{\tilde{\Omega}} \frac{\vec{y}}{\sinh \Theta(\vec{z}, \vec{y})} g(\Theta(\vec{z}, \vec{y})) \tilde{u}_{1}^{2} \mathrm{~d} \tilde{V}
$$

where the integration variable is $\vec{y}$. We write $\Pi: \tilde{\mathbb{H}}^{n} \rightarrow \mathbb{R}^{n}$ for the projection

$$
\Pi \vec{y}=\left(y_{1}, \ldots, y_{n}\right) \quad \text { for } \vec{y} \in \tilde{\mathbb{H}}^{n} .
$$

Assume that there is a $\vec{z}_{0} \in \tilde{\mathbb{H}}^{n}$ and $\alpha \in \mathbb{R}$ such that

$$
\vec{v}\left(\vec{z}_{0}\right)=\alpha \vec{z}_{0}
$$

Under this assumption we chose $R$ to be a Lorentz transformation that maps $\vec{z}_{0}$ to $(0, \ldots, 0,1)$. Then the $\theta$-coordinate of $R \vec{y}$ is $\Theta\left(\vec{z}_{0}, \vec{y}\right)$ and 
the $\vec{\chi}$-coordinate is $\Pi R \vec{y} / \sinh \Theta\left(\vec{z}_{0}, \vec{y}\right)$, such that

$$
\begin{aligned}
\int_{\tilde{\Omega}} \tilde{P}_{i}(R \vec{y}) \tilde{u}_{1}^{2}(\vec{y}) \mathrm{d} \tilde{V} & =\int_{\tilde{\Omega}} \frac{(\Pi R \vec{y})_{i}}{\sinh \Theta\left(\vec{z}_{0}, \vec{y}\right)} g\left(\Theta\left(\vec{z}_{0}, \vec{y}\right)\right) \tilde{u}_{1}^{2}(\vec{y}) \mathrm{d} \tilde{V} \\
& =\left(\Pi R \vec{v}\left(\vec{z}_{0}\right)\right)_{i} \\
& =\alpha\left(\Pi R \vec{z}_{0}\right)_{i}=0
\end{aligned}
$$

Thus it only remains to show that a $\vec{z}_{0}$ exists that meets condition (32). The projection $\Pi$ has a well defined inverse

$$
\Pi^{-1} \vec{\xi}=\left(\xi_{1}, \ldots, \xi_{n}, \sqrt{1+\xi_{1}^{2}+\cdots+\xi_{n}^{2}}\right) \text { for } \vec{\xi} \in \mathbb{R}^{n} .
$$

Also $\Pi \tilde{\Omega} \subset \mathbb{R}^{n}$ is a bounded domain and we can chose a ball $B_{R} \subset \mathbb{R}^{n}$, centered at the origin and of Euclidean radius $R$, such that $\Pi \tilde{\Omega}$ is contained in $B_{R}$. On $B_{R}$ we define the vector field $\vec{w}: B_{R} \rightarrow \mathbb{R}^{n}$ with

$$
\vec{w}(\vec{\xi})=\Pi\left(\vec{v}\left(\Pi^{-1} \vec{\xi}\right)-\frac{\left[\vec{v}\left(\Pi^{-1} \vec{\xi}\right)\right]_{n+1}}{\left[\Pi^{-1} \vec{\xi}\right]_{n+1}} \Pi^{-1} \vec{\xi}\right) .
$$

The set $\Pi^{-1} B_{R}$ and the origin of $\mathbb{R}_{1}^{n}$ span the conus

$$
C=\left\{\vec{y} \in \mathbb{R}_{1}^{n}: y_{n+1} \geq 0 \text { and } \frac{y_{1}^{2}+\cdots+y_{n}^{2}}{y_{n+1}^{2}} \leq \frac{R^{2}}{R^{2}+1}\right\}
$$

and $\tilde{\Omega}$ is contained in $C$. Therefore also $\vec{v}(\vec{z})$, being an integral over vectors in $\tilde{\Omega}$ with positive coefficients, lies in this conus for every $\vec{z} \in$ $\tilde{\mathbb{H}}^{n}$. Thus

$$
\frac{\|\Pi \vec{v}(\vec{z})\|}{[\vec{v}(\vec{z})]_{n+1}} \leq \frac{R}{\sqrt{R^{2}+1}}
$$

Consequently, for any $\vec{\xi} \in \partial B_{R}$ we have, using first the Cauchy-Schwarz inequality and then (34),

$$
\begin{aligned}
\vec{w}(\vec{\xi}) \cdot \vec{\xi} & =\vec{v}\left(\Pi^{-1} \vec{\xi}\right) \cdot \vec{\xi}-\frac{\left[\vec{v}\left(\Pi^{-1} \vec{\xi}\right)\right]_{n+1}}{\left[\Pi^{-1} \vec{\xi}\right]_{n+1}}\|\vec{\xi}\|^{2} \\
& \leq\left\|\Pi \vec{v}\left(\Pi^{-1} \vec{\xi}\right)\right\| R-\frac{\left[\vec{v}\left(\Pi^{-1} \vec{\xi}\right)\right]_{n+1}}{\sqrt{R^{2}+1}} R^{2} \\
& \leq \frac{\left[\vec{v}\left(\Pi^{-1} \vec{\xi}\right)\right]_{n+1}}{\sqrt{R^{2}+1}} R^{2}-\frac{\left[\vec{v}\left(\Pi^{-1} \vec{\xi}\right)\right]_{n+1}}{\sqrt{R^{2}+1}} R^{2}=0
\end{aligned}
$$

This means that $\vec{w}(\vec{\xi})$ points inward everywhere on $\partial B_{R}$. Then by the Brouwer Fixed Point Theorem (see 23, p. 369, prob. $7 \mathrm{~d}$ )) there must be some point $\vec{\xi}_{0} \in B_{R}$ such that $\vec{w}\left(\vec{\xi}_{0}\right)=0$. We put $\vec{\xi}_{0}$ for $\vec{\xi}$ into (33) and multiply with $\Pi^{-1}$ to get

$$
\vec{v}\left(\Pi^{-1} \vec{\xi}_{0}\right)=\frac{\left[\vec{v}\left(\Pi^{-1} \vec{\xi}_{0}\right)\right]_{n+1}}{\left[\Pi^{-1} \vec{\xi}_{0}\right]_{n+1}} \Pi^{-1} \vec{\xi}_{0}
$$


Thus, setting $\vec{z}_{0}=\Pi^{-1} \vec{\xi}_{0}$ we have proven the existence of a $\vec{z}_{0}$ that meets condition (32).

\section{A monotonicity LEMma}

This section is devoted to the proof of the following lemma that states the monotonicity properties of $B$ and $g$ and is crucial for the proof of the PPW conjecture in $\mathbb{H}^{n}$.

Lemma 7.1 (Monotonicity of $B$ and $g$ ). The function $g(\theta)$ defined in (25) is increasing and the function $B(\theta)$ defined in (27) is decreasing.

Similar to the proceeding in 8 ] we will prove Lemma 7.1] by analyzing the function

$$
q(\theta)=\frac{\theta g^{\prime}(\theta)}{g(\theta)}=\theta\left(\frac{z_{1}^{\prime}}{z_{1}}-\frac{z_{0}^{\prime}}{z_{0}}\right)
$$

which is defined by (35) for $\theta \in(0, \tilde{\theta})$ only, but can be extended to a continuous and differentiable function on the whole interval $[0, \tilde{\theta}]$. We will show that $q(\theta)$ has the properties

$$
\begin{aligned}
q(\theta) & \geq 0 \\
q(\theta) & \leq 1 \text { and } \\
q^{\prime}(\theta) & \leq 0
\end{aligned}
$$

for all $\theta \in[0, \tilde{\theta}]$. From (36), the definition (35) of $q$ and the fact that $g \geq$ 0 we conclude directly that $g^{\prime} \geq 0$ and thus $g$ is increasing. Moreover, by solving (35) for $g^{\prime}$, differentiating both sides of the resulting equation and putting (35) in again, we get

$$
g^{\prime \prime}(\theta)=\frac{g}{\theta^{2}}\left(\theta q^{\prime}+q(q-1)\right)
$$

Thus we can conclude from (36), (37) and (38) that $g^{\prime \prime} \leq 0$. For the function

$$
B(\theta)=g^{\prime}(\theta)^{2}+\frac{\nu g^{2}(\theta)}{\sinh ^{2}(\theta)}
$$

this means that the first summand $g^{\prime}(\theta)^{2}$ is decreasing. But then also the second summand is decreasing: Because $g(0)=0$ and because $g^{\prime}$ is decreasing, we have the estimate

$$
g(\theta)=\int_{0}^{\theta} g^{\prime}(\tau) \mathrm{d} \tau \geq \int_{0}^{\theta} g^{\prime}(\theta) \mathrm{d} \tau=g^{\prime}(\theta) \theta .
$$


Thus we can calculate

$$
\begin{aligned}
\left(\frac{g(\theta)}{\sinh \theta}\right)^{\prime} & =\frac{1}{\sinh \theta}\left(g^{\prime}(\theta)-\frac{g(\theta)}{\tanh \theta}\right) \\
& \leq \frac{1}{\sinh \theta}\left(g^{\prime}(\theta)-\frac{g^{\prime}(\theta) \theta}{\tanh \theta}\right) \\
& =\frac{g^{\prime}(\theta)}{\sinh \theta}(1-\theta / \tanh \theta)
\end{aligned}
$$

This is negative because $g^{\prime}$ is positive and $\theta \geq \tanh \theta$. This means that the second summand in the definition of $B$ also decreases.

Remark. In 8 the function $q$ has been defined as in (35), but with $\sin \theta$ replacing the factor $\theta$. One might wonder whether in our $\mathbb{H}^{n}$ case the function $q$ should be defined rather with a factor $\sinh \theta$ than just $\theta$. Actually this would simplify the Riccati equation for $q$ that we will derive below. It turns out, however, that in this case $q$ would not be smaller than one anymore, destroying our scheme of the proof. We thus have to stick to our definition (35) of $q$ and deal with lengthened calculus below.

We have seen that Lemma 7.1 can be shown by proving the equations (36), (37) and (38), so this is what we will do in the remainder of this section. We begin with some basic properties of $q$. From (35) we can see quickly that

$$
q(0)=1, \quad q^{\prime}(0)=0 \quad \text { and } \quad q(\tilde{\theta})=0 .
$$

Differentiating (35) and replacing the second derivatives of $z_{0}$ and $z_{1}$ according to the differential equations they fulfill (equation (17) for $l=$ $0, \lambda=\lambda_{1}\left(S_{1}\right)$ and $l=1, \lambda=\lambda_{2}\left(S_{1}\right)$, respectively), we derive the Riccati equation

$$
q^{\prime}=\frac{q(1-q)}{\theta}-\nu q \operatorname{coth} \theta+\frac{\nu \theta}{\sinh ^{2} \theta}-\theta \epsilon-2 p q .
$$

Here we have defined

$$
p(\theta)=z_{0}^{\prime}(\theta) / z_{0}(\theta), \quad \epsilon=\lambda_{2}-\lambda_{1} \quad \text { and } \quad \nu=n-1 .
$$

We can also establish a Riccati equation for $p$ in a similar fashion as we derived (41). The result is

$$
p^{\prime}=-p^{2}-\nu p \operatorname{coth} \theta-\lambda_{1} \text {. }
$$

Now we set

$$
T(\theta, y):=\frac{y(1-y)}{\theta}-\nu y \operatorname{coth} \theta+\frac{\nu \theta}{\sinh ^{2} \theta}-\theta \epsilon-2 p(\theta) y,
$$

for $y \in \mathbb{R}$ and $\theta \in(0, \tilde{\theta})$. Then by (41) we have

$$
q^{\prime}(\theta)=T(\theta, q(\theta)) \text { for all } \theta \in(0, \tilde{\theta}) .
$$


We call $T^{\prime}$ the derivative of $T$ by $\theta$ and calculate

$$
T^{\prime}(\theta, y)=\frac{y^{2}-y}{\theta^{2}}+\frac{(y+1) \nu}{\sinh ^{2} \theta}-\epsilon-\frac{2 \nu \theta \cosh \theta}{\sinh ^{3} \theta}-2 p^{\prime}(\theta) y .
$$

We are interested in the behavior of $T^{\prime}$ at points $(\theta, y)$ where $T=0$.

Thus we first use (43) to calculate the value of $p$ at such points:

$$
p_{T=0}=\frac{1}{2 y}\left(\frac{y(1-y)}{\theta}-\nu y \operatorname{coth} \theta-\theta \epsilon+\frac{\nu \theta}{\sinh ^{2} \theta}\right) .
$$

Then we put the Riccati equation (42) for $p^{\prime}$ into (45) and finally eliminate $p$ with the help of (46). The result is

$$
\begin{aligned}
T_{T=0}^{\prime}= & \frac{y^{2}-y}{\theta^{2}}+\frac{(y+1) \nu}{\sinh ^{2} \theta}-\epsilon-\frac{2 \nu \theta \cosh \theta}{\sinh ^{3} \theta}+2 y \lambda_{1} \\
& +\frac{1}{2 y}\left(\frac{y-y^{2}}{\theta}-y \nu \operatorname{coth} \theta-\epsilon \theta+\frac{\nu \theta}{\sinh ^{2} \theta}\right)^{2} \\
& +\nu \operatorname{coth} \theta\left(\frac{y-y^{2}}{\theta}-y \nu \operatorname{coth} \theta-\epsilon \theta+\frac{\nu \theta}{\sinh ^{2} \theta}\right) .
\end{aligned}
$$

The right hand side of (47) we be called $Z_{y}(\theta)$. The above analysis holds for all $\theta \in(0, \tilde{\theta})$. For $y=1$ the limits of $Z_{y}$ and $T^{\prime}$ as $\theta \rightarrow 0$ are finite and one can calculate from (42), (45) and (47) that

$$
\begin{aligned}
p^{\prime}(0) & =\lim _{\theta \rightarrow 0} p^{\prime}(\theta)=-\frac{\lambda_{1}}{\nu+1} \\
T^{\prime}(0,1) & =\lim _{\theta \rightarrow 0} T^{\prime}(\theta, 1)=-\lambda_{2}+\left(1+\frac{2}{\nu+1}\right) \lambda_{1}-\frac{2}{3} \nu \\
Z_{1}(0) & =\lim _{\theta \rightarrow 0} Z_{1}(\theta)=(\nu+1)\left(-\lambda_{2}+\left(1+\frac{2}{\nu+1}\right) \lambda_{1}-\frac{2}{3} \nu\right) .
\end{aligned}
$$

Altogether this means that

$$
\begin{array}{ll}
\left.T^{\prime}(\theta, y)\right|_{T=0} & =Z_{y}(\theta) \quad \text { for } \theta \in(0, \tilde{\theta}) \text { and } y \in \mathbb{R}, \\
T^{\prime}(0,1) & =(\nu+1)^{-1} Z_{1}(0) .
\end{array}
$$

The analysis of $Z_{y}$ is somewhat lengthy and will be postponed to Section 8 . The information that we need about $Z_{y}$ is:

Lemma 7.2 (Properties of $Z_{y}$ ).

a) There is no pair $\theta, y$ with $\theta \in(0, \tilde{\theta})$ and $0<y<1$ such that $Z_{y}^{\prime}(\theta)=0$ and $Z_{y}^{\prime \prime}(\theta) \leq 0$.

b) The function $Z_{1}(\theta)$ is strictly increasing in $\theta$ on the interval $(0, \tilde{\theta})$.

Lemma 7.2 will be proven in Section 8 . Now we have all information at hand to prove the equations (36), (37) and (38).

Fact 7.1. The function $q(\theta)$ is non-negative on the interval $[0, \tilde{\theta}]$. 
Proof. To prove (36) we assume the contrary, i.e., that $q$ drops below zero somewhere on $[0, \tilde{\theta}]$. Then there are two points $0<\theta_{1}<\theta_{2} \leq \tilde{\theta}$ with $q\left(\theta_{1}\right)=q\left(\theta_{2}\right)=0$ and $q^{\prime}\left(\theta_{1}\right) \leq 0 \leq q^{\prime}\left(\theta_{2}\right)$.

Suppose first that $\theta_{2}<\tilde{\theta}$. By (41) we have

$$
\left.q^{\prime}\right|_{q=0}=\frac{\nu \theta}{\sinh ^{2} \theta}-\epsilon \theta \quad \text { for } \theta<\tilde{\theta} .
$$

This is a strictly decreasing function in $\theta$ and therefore a contradiction to $q^{\prime}\left(\theta_{1}\right) \leq q^{\prime}\left(\theta_{2}\right)$.

Second, we assume $\theta_{2}=\tilde{\theta}$. Again from (41) we get

$$
3 \lim _{\theta \rightarrow \tilde{\theta}} q^{\prime}(\theta)=\frac{\nu \tilde{\theta}}{\sinh ^{2} \tilde{\theta}}-\epsilon \tilde{\theta} .
$$

We thus get the chain of (in)equalities

$$
0 \geq q^{\prime}\left(\theta_{1}\right)=\frac{\nu \theta_{1}}{\sinh ^{2} \theta_{1}}-\epsilon \theta_{1}>\frac{\nu \tilde{\theta}}{\sinh ^{2} \tilde{\theta}}-\epsilon \tilde{\theta}=3 q^{\prime}(\tilde{\theta}) \geq 0,
$$

again a contradiction.

Fact 7.2. For every small enough $\theta>0$ holds $q(\theta)<1$.

Proof. We recall that $q(0)=1$ and $T(0,1)=0$. Because $Z_{1}$ is strictly increasing by Lemma 7.2 and because $\left.T^{\prime}(\theta, 1)\right|_{T=0}=Z_{1}(\theta)$, it is impossible that $T(\theta, 1)$ is equal to zero on a finite interval. Thus we can limit our consideration to two separate cases:

First, assume $T(\theta, 1)<0$ for all $\theta \in\left(0, \theta_{1}\right)$, where $\theta_{1}$ is some small positive number. Differentiating (43) yields

$$
\left.\frac{\partial T}{\partial y}\right|_{y=1}=-\frac{1}{\theta}-\nu \operatorname{coth} \theta-2 p(\theta) .
$$

Because $p(\theta) \rightarrow 0$ for $\theta \rightarrow 0$, there is some $\theta_{2}>0$ such that

$$
\left.\frac{\partial T}{\partial y}\right|_{y=1}<0 \text { for } \theta \in\left(0, \theta_{2}\right)
$$

In the definition (43) of $T$ we see that $T(\theta, y)$, viewed as a function of $y$ for some fixed $\theta$, is just a parabola opening downwards. We conclude that $T(\theta, y)$ is strictly negative on the set $\left(0, \min \left(\theta_{1}, \theta_{2}\right)\right) \times[1, \infty)$ in the $(\theta, y)$-plane. Keeping in mind (44), this means that $q(\theta)$ must be smaller than one for small enough $\theta>0$.

Second, assume $T(\theta, 1)>0$ for small enough $\theta$. Then $T^{\prime}(0,1) \geq 0$, which means in view of (48) that $Z_{1}(0) \geq 0$. By part b) of Lemma 7.2 , the function $Z_{1}$ is strictly increasing, such that $Z_{1}(\theta)>0$ for all $\theta \in$ $(0, \tilde{\theta})$. We conclude that $T(\theta, 1)$ is strictly positive for all $\theta \in(0, \tilde{\theta})$, because otherwise we would have $Z_{1}(\theta)=T^{\prime}(\theta, 1) \leq 0$ at the point where $T(\theta, 1)$ crosses zero. Now assume that Fact [7.2] is not true, i.e., $q(\theta) \geq 1$ for some $\theta>0$. Then, to meet the condition $q(\tilde{\theta})=0$, there 
must be some $\theta_{1} \in(0, \tilde{\theta})$ with $q\left(\theta_{1}\right)=1$ and $q^{\prime}\left(\theta_{1}\right) \leq 0$. But this would mean that $T\left(\theta_{1}, 1\right) \leq 0$, a contradiction to what was said above.

Fact 7.3. If $0<q(\theta)<1$ for some $\theta \in(0, \tilde{\theta})$, then $q^{\prime}(\theta) \leq 0$.

Proof. Assume the contrary. Then, because of the boundary behavior (40) of $q$, there must be three points $0<\theta_{1}<\theta_{2}<\theta_{3}<\tilde{\theta}$ such that $0<q\left(\theta_{1}\right)=q\left(\theta_{2}\right)=q\left(\theta_{3}\right)=: \tilde{q}<1$ and $q^{\prime}\left(\theta_{1}\right)<0, q^{\prime}\left(\theta_{2}\right)>0$ and $q^{\prime}\left(\theta_{3}\right)<0$. For the function $T(\theta, y)$ this means that

$$
T\left(\theta_{1}, \tilde{q}\right)<0, \quad T\left(\theta_{2}, \tilde{q}\right)>0, \quad T\left(\theta_{4}, \tilde{q}\right)<0 .
$$

We also have the boundary behavior

$$
\begin{aligned}
& \lim _{\theta \rightarrow 0} T(\theta, \tilde{q})=+\infty, \\
& \lim _{\theta \rightarrow \tilde{\theta}} T(\theta, \tilde{q})=+\infty .
\end{aligned}
$$

We conclude that $T(\theta, \tilde{q})$ changes its sign at least four times on the interval $(0, \tilde{\theta})$. Consequently, there are four points $\tilde{\theta}_{1}<\tilde{\theta}_{2}<\tilde{\theta}_{3}<\tilde{\theta}_{4}$ with

$$
Z_{\tilde{q}}\left(\tilde{\theta}_{1}\right) \leq 0, \quad Z_{\tilde{q}}\left(\tilde{\theta}_{2}\right) \geq 0, \quad Z_{\tilde{q}}\left(\tilde{\theta}_{3}\right) \leq 0, \quad Z_{\tilde{q}}\left(\tilde{\theta}_{3}\right) \geq 0 .
$$

Then between $\tilde{\theta}_{1}$ and $\tilde{\theta}_{3}$ there must be some point $\theta$ such that $q^{\prime}(\theta)=0$ and $q^{\prime \prime}(\theta) \leq 0$, a contradiction to part a) of Lemma 7.2 .

From Fact 7.1, Fact 7.2 and Fact 7.3 we conclude that the equations (37) and (38) are true, which proves Lemma 7.1 .

\section{AnAlysis OF THE FUnCTION $Z_{y}$}

This section will be devoted to the proof of Lemma [7.2. Recall that $Z_{y}$ was defined to be the right hand side of (47). It is straight-forward to simplify this expression to

$$
Z_{y}=\sum_{i=1}^{6} c_{i} A_{i}+c_{7}
$$

with

$$
\begin{array}{ll}
A_{1}=-\operatorname{coth}^{2} \theta, & A_{2}=-\theta^{-2}, \\
A_{3}=\theta^{2} \sinh ^{-4} \theta, & A_{4}=-\theta^{2} \sinh ^{-2} \theta, \\
A_{5}=-(\theta \operatorname{coth} \theta-1) \sinh ^{-2} \theta, & A_{6}=\theta^{2}
\end{array}
$$

and

$$
\begin{aligned}
c_{1} & =\frac{1}{2} \nu^{2} y, & c_{2} & =\frac{1}{2}\left(y-y^{3}\right), \\
c_{3} & =\frac{1}{2} \nu^{2} y^{-1}, & c_{4} & =\epsilon \nu y^{-1}, \\
c_{5} & =2 \nu, & c_{6} & =\frac{1}{2} \epsilon^{2} y^{-1} .
\end{aligned}
$$

The constant $c_{7}$ includes all terms that do not depend on $\theta$. Thus none of the $c_{i}$ depends on $\theta$ and

$$
c_{i}>0 \text { for } i=1, \ldots, 6 \text { and } 0<y<1 .
$$


Proof of Lemma 7.2 part a). To prove part a) of Lemma 7.2 we will show that for $0<y<1$ there is no $\theta>0$ such that $Z_{y}^{\prime}(\theta) \leq 0$ and $Z_{y}^{\prime \prime}(\theta) \leq 0$. We will think of $\theta$ as some fixed number and thus drop it for the sake of brevity wherever it appears as an argument of the functions $Z_{y}(\theta)$ or $A_{i}(\theta)$. Further, we consider $\vec{Z}=\left(Z_{y}^{\prime}, Z_{y}^{\prime \prime}\right)$ and $\vec{A}_{i}=\left(A_{i}^{\prime}, A_{i}^{\prime \prime}\right)$ as vectors in the plane. Then we have to show that $\vec{Z}$ does not lie in the third quadrant. It is clear that $\vec{Z}$ is a linear combination with positive coefficients of the $\vec{A}_{i}$. The vector $\vec{A}_{1}$ lies in the fourth quadrant because $A_{1}^{\prime}>0$ and $A_{1}^{\prime \prime}<0$. Let $H$ be the half-plane that is limited by $\left\{c \cdot \overrightarrow{A_{1}}, c \in \mathbb{R}\right\}$ and contains the first quadrant. By the Lemma 8.1 below we see that the vectors $\vec{A}_{i}, i=2, \ldots, 6$ lie in the interior of $H$. We conclude that no linear combinations of the $\overrightarrow{A_{i}}$ with positive coefficients can lie in the third quadrant, which proves Lemma 7.2 .

Lemma 8.1. All cross products

$$
\overrightarrow{A_{1}} \times \overrightarrow{A_{i}}=A_{1}^{\prime} A_{i}^{\prime \prime}-A_{1}^{\prime \prime} A_{i}^{\prime} \quad(i=2, \ldots, 6)
$$

are strictly positive.

Proof of Lemma 8.1. For $i=2$ we calculate

$$
\overrightarrow{A_{1}} \times \overrightarrow{A_{2}}=\frac{4}{\theta^{4} \sinh ^{4} \theta} f(\theta)
$$

with

$$
\begin{aligned}
f(\theta) & =3 \theta \cosh ^{2} \theta-3 \cosh \theta \sinh \theta-\theta \sinh ^{2} \theta \\
& =\theta \cosh 2 \theta+2 \theta-\frac{3}{2} \sinh 2 \theta .
\end{aligned}
$$

The positivity $f$ (and therefore of $\overrightarrow{A_{1}} \times \overrightarrow{A_{2}}$ ) for $\theta>0$ now follows from $f(0)=f^{\prime}(0)=0$ and

$$
f^{\prime \prime}(\theta)=2 \cosh 2 \theta(2 \theta-\tanh 2 \theta)>0 .
$$

For $i=3$ we have

with

$$
\overrightarrow{A_{1}} \times \overrightarrow{A_{3}}=\frac{4 f(\theta)}{\sinh ^{9} \theta}
$$

$$
f(\theta)=-6 \theta \cosh ^{2} \theta \sinh \theta+\theta \sinh \theta+\left(4 \theta^{2}+1\right) \cosh ^{3} \theta-\cosh \theta .
$$

It is $f(0)=0$ and $f^{\prime}(\theta)=\cosh (\theta) g(\theta)$ with $g(\theta)=-3 \cosh \theta \sinh \theta-12 \theta \sinh ^{2} \theta+2 \theta \cosh ^{2} \theta+\theta+12 \theta^{2} \cosh \theta \sinh \theta$.

One can check that $g(0)=0$ and

$$
\begin{aligned}
g^{\prime}(\theta)= & 4 \sinh \theta(\theta \cosh \theta-\sinh \theta)+12\left(\theta^{2} \cosh ^{2} \theta-\sinh ^{2} \theta\right) \\
& +12 \theta^{2} \sinh ^{2} \theta \\
> & 0
\end{aligned}
$$


Consequently, $g(\theta) \geq 0$ for $\theta \geq 0$ and therefore $f^{\prime}(\theta)>0$. It follows that $f(\theta)>0$ for $\theta>0$ and finally $\overrightarrow{A_{1}} \times \overrightarrow{A_{3}}>0$.

For $i=4$ we have

$$
\begin{aligned}
\vec{A}_{1} \times \vec{A}_{4} & =\frac{4}{\sinh ^{6} \theta}\left(2 \theta \cosh ^{2} \theta-\cosh \theta \sinh \theta-\theta\right) \\
& =\frac{4}{\sinh ^{6} \theta}\left(\theta\left(\cosh ^{2} \theta-1\right)+\cosh \theta(\theta \cosh \theta-\sinh \theta)\right) \\
& >0 .
\end{aligned}
$$

For $i=5$ we have

$$
\overrightarrow{A_{1}} \times \overrightarrow{A_{5}}=\frac{2}{\sinh ^{8} \theta} f(\theta)
$$

with

$$
\begin{aligned}
f(\theta) & =-4 \theta \cosh ^{2} \theta+2 \sinh \theta \cosh ^{3} \theta+\sinh \theta \cosh \theta+\theta \\
& =-\theta-2 \theta \cosh 2 \theta+\sinh 2 \theta+\frac{1}{4} \sinh 4 \theta
\end{aligned}
$$

We have $f(0)=f^{\prime}(0)=0$ and

$$
f^{\prime \prime}(\theta)=4 \sinh 2 \theta(\cosh 2 \theta-1)+4 \cosh 2 \theta(\sinh 2 \theta-2 \theta)>0
$$

for $\theta>0$. We conclude that $f(\theta)>0$ for $\theta>0$ and therefore $\overrightarrow{A_{1}} \times \overrightarrow{A_{5}}>$ 0 .

The statement for $i=6$ follows from the fact that $\vec{A}_{6}$ lies in the first quadrant $\left(A_{6}^{\prime}>0, A_{6}^{\prime \prime}>0\right)$ and $\overrightarrow{A_{1}}$ lies in the fourth quadrant.

Proof of Lemma 7.2 part b). We need to show that the function $Z_{1}(\theta)$ as given by (49) is strictly increasing in $\theta$. We start with the partial derivative of $Z_{1}(\theta)$ by $\epsilon$,

$$
\frac{\partial Z_{1}}{\partial \epsilon}=\epsilon \theta^{2}-\frac{\nu \theta^{2}}{\sinh ^{2} \theta}+\frac{\partial c_{7}}{\partial \epsilon}
$$

We note that the right hand side of (50) is an increasing function in $\theta$ and conclude, that if $Z_{1}(\theta)$ is increasing for $\epsilon=0$, then it will be increasing for any $\epsilon>0$. So it only remains to show that

$$
\left.Z_{1}(\theta)\right|_{\epsilon=0}=2 \nu \frac{1-\theta \operatorname{coth} \theta}{\sinh ^{2} \theta}+\frac{\nu^{2}}{2}\left(\frac{\theta^{2}}{\sinh ^{4} \theta}-\operatorname{coth}^{2} \theta\right)+c_{7}
$$

is increasing in $\theta$. We set

$$
f_{1}(\theta)=\frac{1-\theta \operatorname{coth} \theta}{\sinh ^{2} \theta}
$$

and

$$
f_{2}(\theta)=\frac{\theta^{2}}{\sinh ^{4} \theta}-\operatorname{coth}^{2} \theta
$$

and show that $f_{1}$ and $f_{2}$ are increasing separately. 
First,

$$
\begin{aligned}
f_{1}^{\prime}(\theta) & =\sinh ^{-4} \theta\left(-\frac{3}{2} \sinh 2 \theta+2 \theta+\theta \cosh 2 \theta\right) \\
& =\sinh ^{-4} \theta\left(2 \theta+\sum_{j=1,3,5, \ldots} \frac{-3 / 2}{j !}(2 \theta)^{j}+\frac{j / 2}{j !}(2 \theta)^{j}\right)
\end{aligned}
$$

In the sum over $j$ each single term is non-negative for $j \geq 3$. For $j=1$ the terms in the sum cancel the $2 \theta$. We thus have $f_{1}^{\prime}(\theta)>0$ for $\theta>0$, such that $f_{1}$ is strictly increasing.

Second,

$$
\begin{aligned}
f_{2}^{\prime}(\theta) & =\frac{2 \cosh \theta}{\sinh ^{5} \theta}\left(\theta \tanh \theta+\sinh ^{2} \theta-2 \theta^{2}\right) \\
& \geq \frac{2 \cosh \theta}{\sinh ^{5} \theta}\left(\theta\left(\theta-\theta^{3} / 3\right)+\left(\theta+\theta^{3} / 6\right)^{2}-2 \theta^{2}\right) \\
& >0
\end{aligned}
$$

for $\theta>0$, which means that $f_{2}$ also is a strictly increasing function.

\section{Chiti's COMPARISON ARGUMENT IN $\mathbb{H}^{n}$}

Lemma 9.1 which we will state in this section gives the justification for the last step in each of the chains of inequalities (28) and (29).

Let $\Omega^{\star}$ be the spherical rearrangement of $\Omega$, i.e., the geodesic ball (centered at the origin of our coordinate system) with the same $\mathbb{H}^{n}$ volume as $\Omega$. For any function $f: \Omega \rightarrow \mathbb{R}^{+}$we define the decreasing rearrangement $f^{\sharp}(s)$ and the spherical decreasing rearrangement $f^{\star}(\theta, \vec{\chi})=f^{\star}(\theta)$. The former is a decreasing function from $[0,|\Omega|]$ to $\mathbb{R}^{+}$and is equimeasurable with $f$ in the measure of $\mathbb{H}^{n}$. The function $f^{\star}$ is defined on $\Omega^{\star}$, spherically symmetric, equimeasurable with $f$ and decreasing in $\theta$. The functions $f^{\sharp}$ and $f^{\star}$ are tightly connected by

$$
f^{\star}(\theta, \vec{\chi})=f^{\sharp}(A(\theta)),
$$

where

$$
A(\theta)=n C_{n} \int_{0}^{\theta} \sinh ^{n-1} \theta^{\prime} \mathrm{d} \theta^{\prime}
$$

is the volume of a geodesic ball in $\mathbb{H}^{n}$ with the radius $\theta$. Here $n C_{n}$ is the surface area of the $(n-1)$-dimensional unit sphere in Euclidean space. In a completely analogous way we also define the increasing rearrangements $f_{\sharp}$ and $f_{\star}$.

Lemma 9.1 (Chiti comparison result). Let $u_{1}(\theta, \vec{\chi})$ be the first Dirichlet eigenfunction of $-\Delta$ on $\Omega$ and $z_{0}(\theta)$ the first Dirichlet eigenfunction of $-\Delta$ on $S_{1}$, normalized such that

$$
\int_{\Omega} u_{1}^{2} \mathrm{~d} V=\int_{S_{1}} z_{0}^{2} \mathrm{~d} V .
$$


Then there is some $\theta_{0} \in(0, \tilde{\theta})$ such that

$$
\begin{array}{ll}
z_{0}(\theta) \geq u_{1}^{\star}(\theta) & \text { for } \theta \in\left(0, \theta_{0}\right) \text { and } \\
z_{0}(\theta) \leq u_{i}^{\star}(\theta) & \text { for } \theta \in\left(\theta_{0}, \tilde{\theta}\right) .
\end{array}
$$

Proof. Define $\Omega_{t}=\left\{x \in \Omega \mid u_{1}(x)>t\right\}$ and $\partial \Omega_{t}=\left\{x \in \Omega \mid u_{1}(x)=t\right\}$. Let $\mu(t)=\left|\Omega_{t}\right|$ and $\left|\partial \Omega_{t}\right|=H_{n-1}\left(\partial \Omega_{t}\right)$, where $H_{n-1}$ denotes the $(n-1)$ dimensional measure on $\mathbb{H}^{n}$. Then the co-area formula

$$
-\mu^{\prime}(t)=\int_{\partial \Omega_{t}} \frac{1}{\left|\operatorname{Grad} u_{1}\right|} \mathrm{d} H_{n-1}
$$

holds (see, e.g., [13], p. 86). On the other hand, applying Gauss' Theorem (in its form for Riemannian manifolds, see, e.g., [13, p. 7) to $-\Delta u_{1}=\lambda_{1} u_{1}$ yields

$$
\int_{\Omega_{t}} \lambda_{1} u_{1} \mathrm{~d} V=\int_{\partial \Omega_{t}}\left|\operatorname{Grad} u_{1}\right| \mathrm{d} H_{n-1}
$$

since the outward normal to $\Omega_{t}$ is $-\operatorname{Grad} u_{1} /\left|\operatorname{Grad} u_{1}\right|$. Using the Cauchy-Schwarz inequality and equations (55) and (56) we get

$$
\left(H_{n-1}\left(\partial \Omega_{t}\right)\right)^{2}=\left(\int_{\partial \Omega_{t}} \mathrm{~d} H_{n-1}\right)^{2} \leq-\mu^{\prime}(t) \lambda_{1} \int_{\Omega_{t}} u_{1} \mathrm{~d} V .
$$

In $\mathbb{H}^{n}$ the classical isoperimetric inequality holds true [13, 24], i.e.,

$$
H_{n-1}\left(\partial \Omega_{t}\right) \geq H_{n-1}\left(\partial\left(\Omega_{t}^{\star}\right)\right) .
$$

Recall the definition (53) and write $A^{-1}$ for the inverse function of $A$. Then the $(n-1)$-dimensional measure of $\partial\left(\Omega_{t}^{\star}\right)$ can in turn be expressed by

$$
H_{n-1}\left(\partial\left(\Omega_{t}^{\star}\right)\right)=n C_{n} \sinh ^{n-1} \theta A^{-1}\left(\left|\Omega_{t}^{\star}\right|\right)=A^{\prime}\left(A^{-1}\left(\left|\Omega_{t}^{\star}\right|\right)\right) .
$$

Hence, equation (58) turns into

$$
H_{n-1}\left(\partial \Omega_{t}\right) \geq A^{\prime}\left(A^{-1}\left(\left|\Omega_{t}^{\star}\right|\right)\right)
$$

and (57) can be written as

$$
\lambda_{1} \int_{\Omega_{t}} u_{1} \mathrm{~d} V \geq-\frac{1}{\mu^{\prime}(t)} A^{\prime}\left(A^{-1}\left(\left|\Omega_{t}^{\star}\right|\right)\right)^{2} .
$$

Finally we use the fact that

$$
\int_{\Omega_{t}} u_{1} \mathrm{~d} V=\int_{0}^{\mu(t)} u_{1}^{\sharp}(s) \mathrm{d} s,
$$

which follows directly from the definition of $u_{1}^{\sharp}$ above. Since $u_{1}^{\sharp}(s)$ is the inverse function to $\mu(t)$, we have

$$
-\frac{\mathrm{d} u_{1}^{\sharp}}{\mathrm{d} s}=-\frac{1}{\mu^{\prime}(t)},
$$


which combined with (61) and (62) yields

$$
-\frac{\mathrm{d} u_{1}^{\sharp}}{\mathrm{d} s} \leq \lambda_{1} A^{\prime}\left(A^{-1}(s)\right)^{-2} \int_{0}^{s} u_{1}^{\sharp}\left(s^{\prime}\right) \mathrm{d} s^{\prime} .
$$

One can check that equality holds in all the steps leading to (63), if one replaces $\Omega$ by $S_{1}$ and $u_{1}$ by $z_{0}$. Therefore,

$$
-\frac{\mathrm{d} z_{0}^{\sharp}}{\mathrm{d} s}=\lambda_{1} A^{\prime}\left(A^{-1}(s)\right)^{-2} \int_{0}^{s} z_{0}^{\sharp}\left(s^{\prime}\right) \mathrm{d} s^{\prime} .
$$

Using the relations (63) and (64) and keeping in mind the normalization (54), we will prove that the functions $u_{1}^{\sharp}$ and $z_{0}^{\sharp}$ are either identical or they cross each other exactly once on the interval $\left[0,\left|S_{1}\right|\right]$. The arguments we use depend on the fact that $u_{1}^{\sharp}$ and $z_{0}^{\sharp}$ are continuous functions. By definition of the rearrangement, both functions are decreasing and we have $z_{0}^{\sharp}\left(\left|S_{1}\right|\right)=u_{1}^{\sharp}(|\Omega|)=0$. Recall that from the Rayleigh-Faber-Krahn inequality and from $\lambda_{1}\left(S_{1}\right)=\lambda_{1}(\Omega)$ follows that $\left|S_{1}\right| \leq|\Omega|$. From the normalization (54) it is clear that $z_{0}^{\sharp}$ and $u_{1}^{\sharp}$ are either identical or cross at least once on $\left[0,\left|S_{1}\right|\right]$. To show that they cross exactly once, we assume the contrary, i.e., that they cross at least twice. Then there are two points $0 \leq s_{1}<s_{2}<\left|S_{1}\right|$ such that $u_{1}^{\sharp}(s)>z_{0}^{\sharp}(s)$ for $s \in\left(s_{1}, s_{2}\right), u_{1}^{\sharp}\left(s_{2}\right)=z_{0}^{\sharp}\left(s_{2}\right)$ and either $u_{1}^{\sharp}\left(s_{1}\right)=z_{0}^{\sharp}\left(s_{1}\right)$ or $s_{1}=0$. We set

$$
v(s)= \begin{cases}u_{1}^{\sharp}(s) & \text { on }\left[0, s_{1}\right] \text { if } \int_{0}^{s_{1}} u_{1}^{\sharp}(s) \mathrm{d} s>\int_{0}^{s_{1}} z_{0}^{\sharp}(s) \mathrm{d} s, \\ z_{0}^{\sharp}(s) & \text { on }\left[0, s_{1}\right] \text { if } \int_{0}^{s_{1}} u_{1}^{\sharp}(s) \mathrm{d} s \leq \int_{0}^{s_{1}} z_{0}^{\sharp}(s) \mathrm{d} s, \\ u_{1}^{\sharp}(s) & \text { on }\left[s_{1}, s_{2}\right], \\ z_{1}^{\sharp}(s) & \text { on }\left[s_{2},\left|S_{1}\right|\right] .\end{cases}
$$

Then one can convince oneself that because of (63) and (64)

$$
-\frac{\mathrm{d} v}{\mathrm{~d} s} \leq \lambda_{1} A^{\prime}\left(A^{-1}(s)\right)^{-2} \int_{0}^{s} v\left(s^{\prime}\right) \mathrm{d} s^{\prime}
$$

for all $s \in\left[0,\left|S_{1}\right|\right]$. Now define the test function $\Psi(\theta, \vec{\chi})=v(A(\theta))$. Using the Rayleigh-Ritz characterization of $\lambda_{1}$, then (66) and finally 
an integration by parts, we get (if $z_{0}$ and $u_{1}$ are not identical)

$$
\begin{aligned}
\lambda_{1} \int_{S_{1}} \Psi^{2} \mathrm{~d} V & <\int_{S_{1}}|\operatorname{Grad} \Psi|^{2} \mathrm{~d} V \\
& =\int_{0}^{\tilde{\theta}}\left(A^{\prime}(\theta) v^{\prime}(A(\theta))\right)^{2} A^{\prime}(\theta) \mathrm{d} \theta \\
& \leq-\int_{0}^{\tilde{\theta}} A^{\prime}(\theta) v^{\prime}(A(\theta)) \lambda_{1} \int_{0}^{A(\theta)} v\left(s^{\prime}\right) \mathrm{d} s^{\prime} \mathrm{d} \theta \\
& =\lambda_{1} \int_{0}^{\left|S_{1}\right|} v(s)^{2} \mathrm{~d} s \\
& =\lambda_{1} \int_{S_{1}} \Psi^{2} \mathrm{~d} V
\end{aligned}
$$

Comparing the first and the last term in the above chain of (in)equalities

reveals a contradiction to our assumption of two intersections of $u_{1}^{\sharp}$ and $z_{0}^{\sharp}$, thus proving Lemma 9.1 .

\section{REFERENCES}

[1] M. S. Ashbaugh and R. D. Benguria, Log-concavity of the ground state of Schrödinger operators: a new proof of the Baumgartner-Grosse-Martin inequality, Phys. Lett. A 131 (1988), 273-276.

[2] M. S. Ashbaugh and R. D. Benguria, Optimal lower bounds for eigenvalue gaps for Schrödinger operators with symmetric single-well potentials and related results, Maximum Principles and Eigenvalue Problems in Partial Differential Equations, P. W. Schaefer, editor, Pitman Research Notes in Mathematics Series, vol. 175, Longman Scientific and Technical, Harlow, Essex, United Kingdom, 1988, pp. 134-145.

[3] M. S. Ashbaugh and R. D. Benguria, Proof of the Payne-Pólya-Weinberger conjecture, Bull. Amer. Math. Soc. 25 (1991), 19-29.

[4] M. S. Ashbaugh and R. D. Benguria, A sharp bound for the ratio of the first two eigenvalues of Dirichlet Laplacians and extensions, Annals of Math. 135 (1992), 601-628.

[5] M. S. Ashbaugh and R. D. Benguria, A second proof of the Payne-PólyaWeinberger conjecture, Commun. Math. Phys. 147 (1992), 181-190.

[6] M. S. Ashbaugh, and R. D. Benguria, Isoperimetric inequalities for eigenvalue ratios, Partial Differential Equations of Elliptic Type, Cortona, 1992, A. Alvino, E. Fabes, and G. Talenti, editors, Symposia Mathematica, vol. 35, Cambridge University Press, Cambridge, United Kingdom, 1994, pp. 1-36.

[7] M. S. Ashbaugh and R. D. Benguria, Sharp upper bound to the first nonzero Neumann eigenvalue for bounded domains in spaces of constant curvature, J. London Math. Soc. (2) 52 (1995), 402-416.

[8] M.S. Ashbaugh, R.D. Benguria: A sharp bound for the ratio of the first two Dirichlet eigenvalues of a domain in a hemisphere of $\mathbb{S}^{n}$, Transactions of the AMS 353 No. 3 (2000), 1055-1087

[9] C. Bandle, Isoperimetric Inequalities and Applications, Pitman Monographs and Studies in Mathematics, vol. 7, Pitman, Boston, 1980.

[10] R.D. Benguria, H. Linde: A second eigenvalue bound for the Dirichlet Schrödinger operator, preprint 
[11] B. Baumgartner, H. Grosse, A. Martin: The Laplacian of the potential and the order of energy levels, Physics Letters 146B No. 5 (1984), 363-366

[12] B. Baumgartner, H. Grosse and A. Martin, Order of levels in potential models, Nucl. Phys. B 254 (1985), 528-542.

[13] I. Chavel: Eigenvalues in Riemannian geometry, Academic Press, Inc. (1984)

[14] G. Chiti, A reverse Hölder inequality for the eigenfunctions of linear second order elliptic operators, J. Appl. Math. and Phys. (ZAMP) 33 (1982), 143-148.

[15] G. Chiti, An isoperimetric inequality for the eigenfunctions of linear second order elliptic operators, Boll. Un. Mat. Ital. (6) 1-A (1982), 145-151.

[16] G. Chiti, A bound for the ratio of the first two eigenvalues of a membrane, SIAM J. Math. Anal. 14 (1983), 1163-1167.

[17] G. Faber, Beweis, dass unter allen homogenen Membranen von gleicher Fläche und gleicher Spannung die kreisförmige den tiefsten Grundton gibt, Sitzungberichte der mathematisch-physikalischen Klasse der Bayerischen Akademie der Wissenschaften zu München Jahrgang, 1923, pp. 169-172.

[18] C. Haile: A second eigenvalue bound for the Dirichlet Schrödinger equation with a radially symmetric potential, Electronic Journal of Differential Equations 2000 No. 10 (2000), 1-19.

[19] E.M. Harrell II, P.L. Michel: Commutator bounds for eigenvalues, with applications to spectral geometry, Commun. Partial Diff. Eqs. 19 (1994), 2037-2055

[20] E. Krahn, Über eine von Rayleigh formulierte Minimaleigenschaft des Kreises, Math. Ann. 94 (1925), 97-100.

[21] E. Krahn, Über Minimaleigenschaften der Kugel in drei und mehr Dimensionen, Acta Comm. Univ. Tartu (Dorpat) A9 (1926), 1-44. [English translation: Minimal properties of the sphere in three and more dimensions, Edgar Krahn 1894-1961: A Centenary Volume, Ü. Lumiste and J. Peetre, editors, IOS Press, Amsterdam, The Netherlands, 1994, pp. 139-174.]

[22] J.M. Luttinger: Generalized isoperimetric inequalities, Proc. Nat. Acad. Sci. USA 70 (1973), 1005-1006

[23] J.R. Munkres: Topology, A first course, Prentice-Hall, Englewood Cliffs, 1975

[24] R. Osserman, The isoperimetric inequality, Bull. Amer. Math. Soc. 84, (1978), $1182-1238$.

[25] L. E. Payne, G. Pólya, and H. F. Weinberger, Sur le quotient de deux fréquences propres consécutives, Comptes Rendus Acad. Sci. Paris 241 (1955), 917-919.

[26] L. E. Payne, G. Pólya, and H. F. Weinberger, On the ratio of consecutive eigenvalues, J. Math. and Phys. 35 (1956), 289-298.

[27] J. W. S. Rayleigh, The Theory of Sound, 2nd. ed. revised and enlarged (in 2 vols.), Dover Publications, New York, 1945 (republication of the 1894/1896 edition).

[28] E. Sperner, Zur Symmetrisierung von Funktionen auf Sphären, Math. Z. 134 (1973), 317-327

[29] G. Talenti: Elliptic equations and rearrangements, Ann. Scuola Norm. Sup. Pisa (4) 3 (1976), 697-718

E-mail address: RBenguri@fis.puc.cl, Helmut.Linde@gmx.de

Department of Physics, Pontificía Universidad Católica de Chile Casilla 306, Correo 22 Santiago, Chile. 